\title{
An Efficient Strategy for Enhancing the Photosensitivity of Photochromic [2.2]Paracyclophane-Bridged Imidazole Dimers
}

\author{
Katsuya Mutoh, Sayaka Hatano and Jiro Abe* \\ Department of Chemistry, School of Science and Engineering, Aoyama Gakuin University, \\ 5-10-1 Fuchinobe, Sagamihara, Kanagawa 229-8558 \\ jiro_abe@chem.aoyama.ac.jp
}

\begin{abstract}
We describe an efficient strategy for designing high-performance fast-photochromic molecules with increased photosensitivity to solar UVA radiation. The photosensitivity of [2.2]paracyclophane-bridged imidazole dimers can be readily enhanced through appropriate design of the phenyl rings attached to the imidazole rings. The diversity of the molecular design and the ease of synthesis make this class of photochromic molecules highly attractive for high-performance ophthalmic plastic lenses.
\end{abstract}

Keywords: photochromism, HABI, bisimidazole, [2.2]paracyclophane

\section{Introduction}

Photochromic molecules change their color upon irradiation with light. The photogenerated state of a photochromic molecule can switch back to the original state either thermally, once irradiation is terminated, or photochemically, upon illumination with a specific wavelength of light. Research into organic photochromic materials is of considerable interest because of their potential application in ophthalmic plastic lenses, high-density optical data storage, optical waveguides, and shutters [1]. These applications take advantage of the fact that the absorbance, fluorescence, and refractive index of a photochromic material can be modulated under the influence of optical stimulations [2]. In particular, thermally reversible photochromic molecules offer the opportunity to change and reset the molecular properties by simply turning a light source on and off. Increasing the thermal-bleaching rate for thermally reversible photochromic molecules is essential for the development of high-performance ophthalmic plastic lenses and revolutionary optical switching devices [3]. We recently developed a photochromic [2.2]PC-bridged imidazole dimer (a)
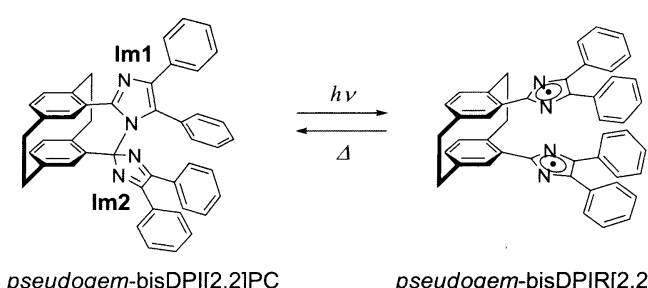

(b)

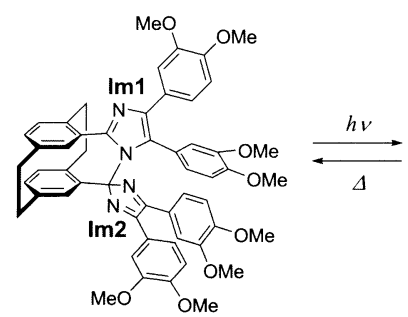

pseudogem-bisTMDPI[2.2]PC

pseudogem-bisTMDPIR[2.2]PC

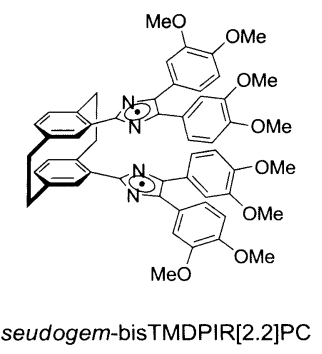

Scheme 1. Photochromic reaction of [2.2]paracyclophane-bridged imidazole dimers.

(Scheme 1a), with a [2.2]paracyclophane ([2.2]PC) moiety that couples two diphenylimidazole (DPI) groups, that shows instantaneous coloration upon exposure to UV light and rapid fading in the dark [4]. In this study, we investigate the structure-property 
relationships based on theoretical calculations and demonstrate an efficient strategy for designing a high-performance fast-photochromic molecule with increased photosensitivity to solar ultraviolet A (UVA) radiation (320-400 nm wavelength).

\section{Experimental}

\subsection{Materials}

All reagents were purchased from Kanto Chemical Co., Inc., Tokyo Chemical Industry Co., Ltd., Wako Pure Chemical Industries, Ltd., Aldrich Chemical Company, Inc, and ACROS Oraganics, and were used without further purification. All reaction solvents were distilled on the appropriate drying reagents prior to use. All reactions were monitored by thin-layer chromatography carried out on $0.2 \mathrm{~mm}$ E. Merck silica gel plates $(60 \mathrm{~F}-254)$. Column chromatography was performed on silica gel (Wakogel $\mathbb{R}$ C-300). 3,3',4,4'-Tetramethoxybenzil was prepared according to a literature procedure [5].

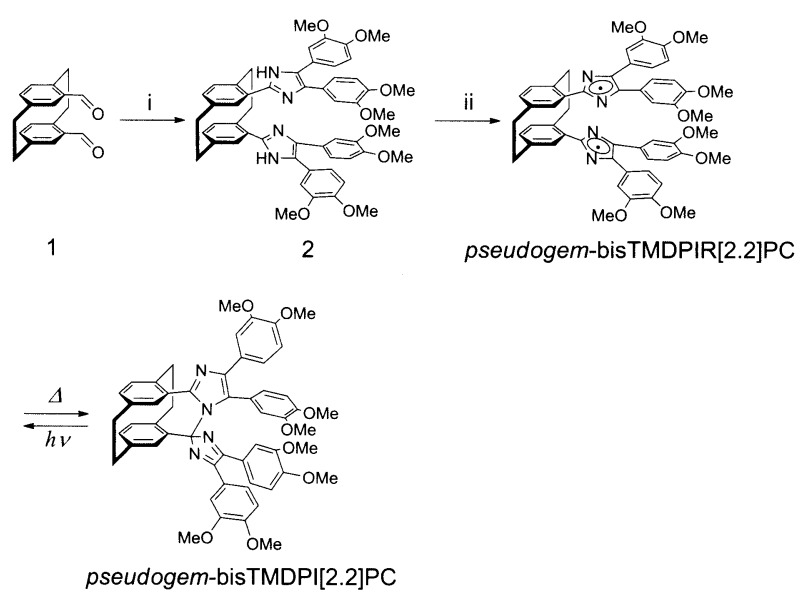

Scheme 2. Synthesis of pseudogem-bisTMDPI[2.2]. (i) 3,3',4,4'-tetramethoxybenzil, $\mathrm{AcONH}_{4}, \mathrm{AcOH}$, reflux, 1 day; (ii) $\mathrm{K}_{3}\left[\mathrm{Fe}(\mathrm{CN})_{6}\right]$ aq., $\mathrm{KOH}$, benzene, r.t., $30 \mathrm{~min}$.

\section{pseudogem-bis(4,5-(3,4,-dimethoxyphenyl)-1H -imidazol-2-yl)[2.2]paracyclophane (2)}

[2.2]Paracyclophane-4,13-dicarbaldehyde [6] (149 mg, $0.565 \mathrm{mmol}$ ), 3,3',4,4'tetramethoxybenzil $(373 \mathrm{mg}, 1.130 \mathrm{mmol})$ and ammonium acetate $(2.6 \mathrm{~g}, 33.73 \mathrm{mmol})$ were refluxed in acetic acid $(22.5 \mathrm{~mL})$ for 1 day. The reaction mixture was cooled with ice bath, and neutralized with aqueous $\mathrm{NH}_{3}$, to form a yellow precipitate. The aqueous layer was extracted with dichloromethane. The combined organic layer was washed with water and brine, dried over $\mathrm{Na}_{2} \mathrm{SO}_{4}$, filtered, and evaporated. The product was purified with silica gel column chromatography twice, using hexane/THF $=1 / 1$ and then $\mathrm{CH}_{2} \mathrm{Cl}_{2}$ /ethyl acetate $=2 / 1$, as eluents respectively, to give a red-brown amorphous solid (2), $166 \mathrm{mg}$ (33.2\%). ${ }^{1} \mathrm{H}-\mathrm{NMR}\left(500 \mathrm{MHz}\right.$, DMSO- $\left.d_{6}\right) \delta: 11.49(\mathrm{~s}, 2 \mathrm{H})$, $7.14(\mathrm{~s}, 2 \mathrm{H}), 6.95(\mathrm{~d}, J=7.9 \mathrm{~Hz}, 2 \mathrm{H}), 6.84(\mathrm{~d}, J=$ $1.8 \mathrm{~Hz}, 2 \mathrm{H}), 6.75-6.57(\mathrm{~m}, 12 \mathrm{H}), 4.55-4.54(\mathrm{~d}, J=$ $7.5 \mathrm{~Hz}, 2 \mathrm{H}), 3.72(\mathrm{~s}, 6 \mathrm{H}), 3.66(\mathrm{~s}, 6 \mathrm{H}), 3.42(\mathrm{~s}, 6 \mathrm{H})$, $3.36(\mathrm{~s}, 6 \mathrm{H}), 3.13-3.04(\mathrm{~m}, 6 \mathrm{H})$; FAB-MS: $\mathrm{m} / \mathrm{z}$ $885[\mathrm{M}+\mathrm{H}]^{+}$.

\section{pseudogem-bisTMDPI[2.2]PC}

All manipulations were carried out with the exclusion of light. Under nitrogen, to a solution of $2(106 \mathrm{mg}, 0.112 \mathrm{mmol})$ in benzene $(10 \mathrm{~mL})$ was added the solution of potassium ferricyanide (1.958 g, $5.947 \mathrm{mmol})$ and $\mathrm{KOH}(0.71 \mathrm{~g}, 12.57$ $\mathrm{mmol})$ in water $(20 \mathrm{~mL})$, and the reaction mixture was vigorously stirred for $30 \mathrm{~min}$. The organic layer was separated, exhaustively washed with water, and concentrated in vacuo. Then the crude product was purified by recrystallization from $\mathrm{CH}_{2} \mathrm{Cl}_{2} /$ hexane to give yellow crystals, $50 \mathrm{mg}$ (1:0.55 mixture of diastereomers $47 \%)$. ${ }^{1} \mathrm{H}$-NMR $\left(500 \mathrm{MHz}, \mathrm{CDCl}_{3}\right)$ mixture of diastereomers $\delta$ : $7.29(\mathrm{~d}, \mathrm{~J}=1.9 \mathrm{~Hz}, 2 \mathrm{H}$, two diastereomers), 7.15 (dd, $\mathrm{J}=7.7,1.9 \mathrm{~Hz}, 2 \mathrm{H}$, two diastereomers), 7.01-6.99 (m, 2H, two diastereomers), 6.96-6.93 $(\mathrm{m}, 2 \mathrm{H}$, two diastereomers), 6.91-6.83 $(\mathrm{m}, 10 \mathrm{H}$, two diastereomers), 6.76-6.74 $(\mathrm{m}, 2 \mathrm{H}$, two diastereomers $), \quad 6.72-6.60 \quad(\mathrm{~m}, \quad 12 \mathrm{H}, \quad$ two diastereomers $), \quad 6.51-6.48 \quad(\mathrm{~m}, \quad 2 \mathrm{H}, \quad$ two diastereomers), 6.45 (dd, $\mathrm{J}=8.0,1.6 \mathrm{~Hz}, 2 \mathrm{H}$, two diastereomers $), \quad 4.65-4.61 \quad(\mathrm{~m}, \quad 1 \mathrm{H}, \quad$ one diastereomer $), \quad 4.55-4.50 \quad(\mathrm{~m}, \quad 1 \mathrm{H}, \quad$ one diastereomer), $3.92(\mathrm{~s}, 3 \mathrm{H}$, one diastereomer), $3.90(\mathrm{~s}, 3 \mathrm{H}$, one diastereomer), $3.89(\mathrm{~s}, 3 \mathrm{H}$, one diastereomer), $3.87(\mathrm{~s}, 3 \mathrm{H}$, one diastereomer), 3.79 (app t, J $=2.6 \mathrm{~Hz}, 15 \mathrm{H}$, two diastereomers), $3.74(\mathrm{~s}, 3 \mathrm{H}$, one diastereomer), $3.69 \mathrm{~s}, 3 \mathrm{H}$, one diastereomer), $3.65(\mathrm{~s}, 3 \mathrm{H}$, one diastereomer), $3.56(\mathrm{~s}, 3 \mathrm{H}$, one diastereomer), $3.55(\mathrm{~s}, 3 \mathrm{H}$, one diastereomer), $3.38-2.92 \quad(\mathrm{~m}, \quad 26 \mathrm{H}$, two diastereomers); FAB-MS: $m / z 883[\mathrm{M}+\mathrm{H}]^{+}$.

\subsection{Laser flash photolysis}

The laser flash photolysis experiments were carried out with a Unisoku TSP-1000 time-resolved spectrophotometer. A Continuum Minilite II Nd:YAG (Q-switched) laser with the third harmonic at $355 \mathrm{~nm}$ (ca. $8 \mathrm{~mJ}$ per $5 \mathrm{~ns}$ pulse) was employed for the excitation light. The 
probe beam from a OSRAM HLX64623 halogen lamp was guided with an optical fiber scope to be arranged in an orientation perpendicular to the exciting laser beam. The probe beam was monitored with a Hamamatsu R2949 photomultiplier tube through a prior to the laser flash photolysis experiments.

\subsection{DFT calculations}

All calculations were carried out using the Gaussian 03 program (Revision E.01) [7]. The molecular structures were fully optimized at the MPW1PW91/6-31G(d) level of the theory, and analytical second derivatives were computed using vibrational analysis to confirm each stationary point to be a minimum. TDDFT calculations were performed at the MPW1PW91/6-31+G(d) level of the theory for the optimized structures.

\section{Results and Discussion}

Pseudogem-bisDPI[2.2]PC

shows

photoinduced homolytic bond cleavage of the $\mathrm{C}-\mathrm{N}$ bond between the imidazole rings similar to hexaarylbiimidazole (HABI) [8], and successive fast $\mathrm{C}-\mathrm{N}$ bond formation. The back-reaction is not accelerated by irradiation with visible light. The half-life of colored species at $25{ }^{\circ} \mathrm{C}$ in benzene is $33 \mathrm{~ms}$. Fast thermal-bleaching kinetics enable a solution color change only where the solution is irradiated with UV light, because the thermal-bleaching rate is much faster than the diffusion rate of the colored species at room temperature. However, no color change is discernible at room temperature under sunlight, owing to the small extinction coefficient around the UVA radiation region. Photosensitivity to sunlight is essential for application to ophthalmic photochromic lenses. Fig. 1(a) shows the UV-vis absorption spectra of pseudogem-bisDPI[2.2]PC measured in acetonitrile solution, along with that obtained by TDDFT calculation (MPW1PW91/ $6-31+\mathrm{G}(\mathrm{d}))$ for the molecular geometry optimized at the MPW1PW91/6-31G(d) level of the theory, indicated by the red perpendicular lines. The first calculated band $\left(\mathrm{S}_{0} \rightarrow \mathrm{S}_{1}\right)$ at $461 \mathrm{~nm}$ is described by a HOMO $\rightarrow$ LUMO transition (Fig. 2(a)). The oscillator strength $(f=0.0004)$ of this transition is almost zero because of the small orbital overlap between these two molecular orbitals (MOs). Pseudogem-bisDPI[2.2]PC has two types of imidazole rings - Im1 and $\operatorname{Im} 2$ - as indicated in Scheme $1 . \operatorname{Im} 1$ is a resonant planar structure that
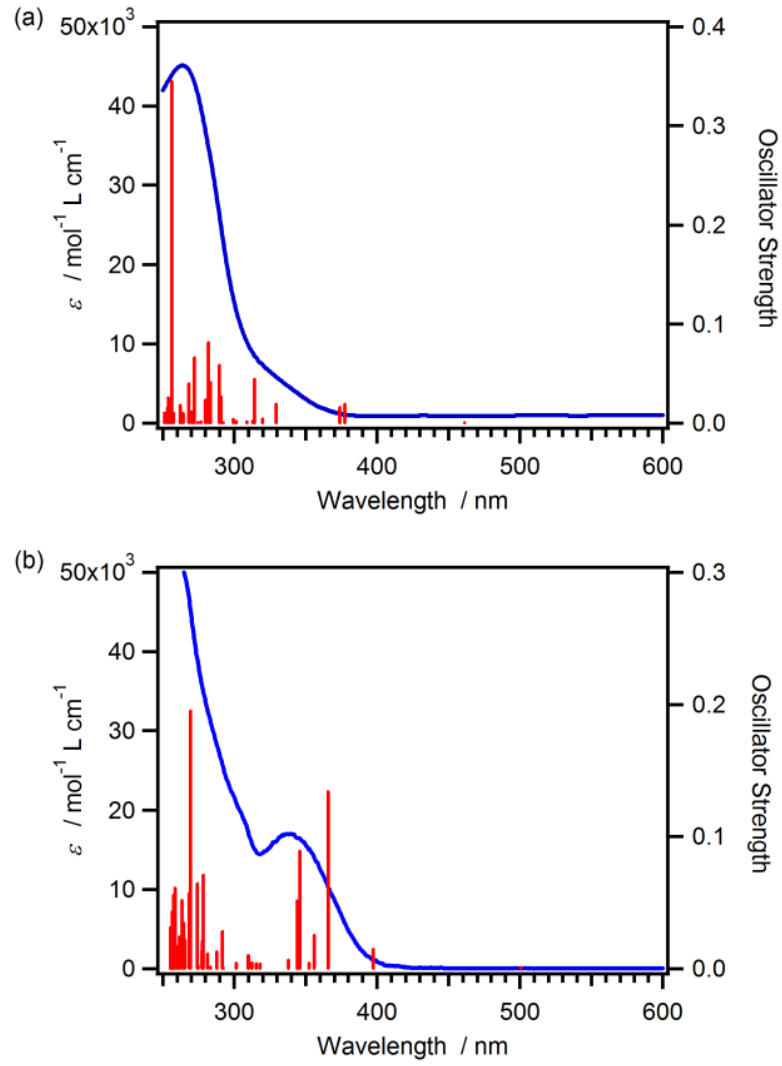

Figure 1. UV-vis absorption spectra of (a) pseudogem-bisDPI[2.2]PC, and pseudogem-bisTMDPI[2.2]PC in acetonitrile at $25^{\circ} \mathrm{C}$. The calculated spectra (MPW1PW91/6-31+ G(d)//MPW1PW91/6-31G(d)) are shown by the red perpendicular lines.

has a typical bond distance for a $6 \pi$ electron system with an electron-donating characteristic, whereas $\operatorname{Im} 2$ has two localized $\mathrm{C}=\mathrm{N}$ double bonds and one $\mathrm{sp}^{3}$ carbon connecting $\operatorname{Im} 1$, consistent with a $4 \pi$-electron system with an electron-withdrawing characteristic. Thus, the first excited state is characterized by the intramolecular charge-transfer (CT) transition from the HOMO delocalized over the electron-donating Im1 to the LUMO delocalized over the electron-withdrawing $\operatorname{Im} 2$, as proved by the TDDFT calculation. The second band $\left(\mathrm{S}_{0} \rightarrow\right.$ $\left.\mathrm{S}_{2}\right)$ at $378 \mathrm{~nm}(f=0.0193)$ is associated with HOMO-1 $\rightarrow$ LUMO and HOMO-2 $\rightarrow$ LUMO transitions. The HOMO-1 and the HOMO-2 are localized on the [2.2]PC moiety, reflecting the well-known electron-donating ability of [2.2]PC. As in the case of the orbital overlap between the HOMO and the LUMO those between the two occupied MOs and the LUMO are also small.

In order to increase photosensitivity to solar UVA radiation, an absorption band with a large 
(a)
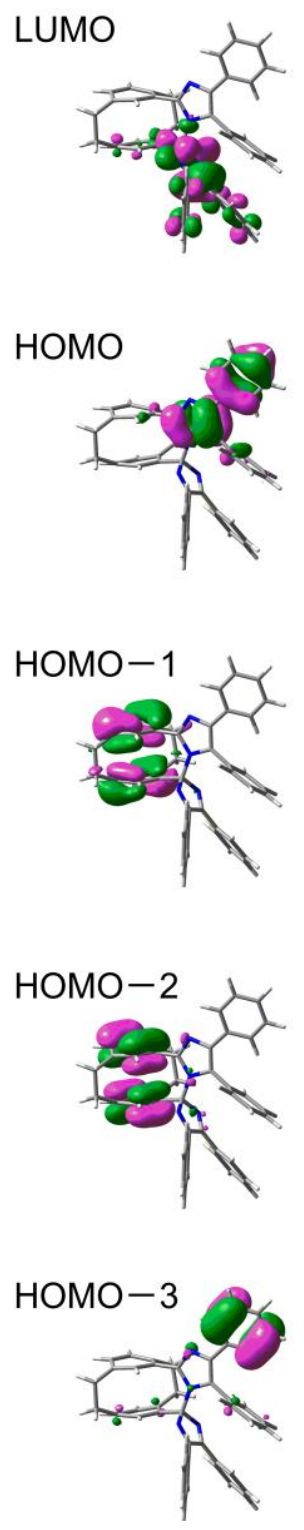

(b)
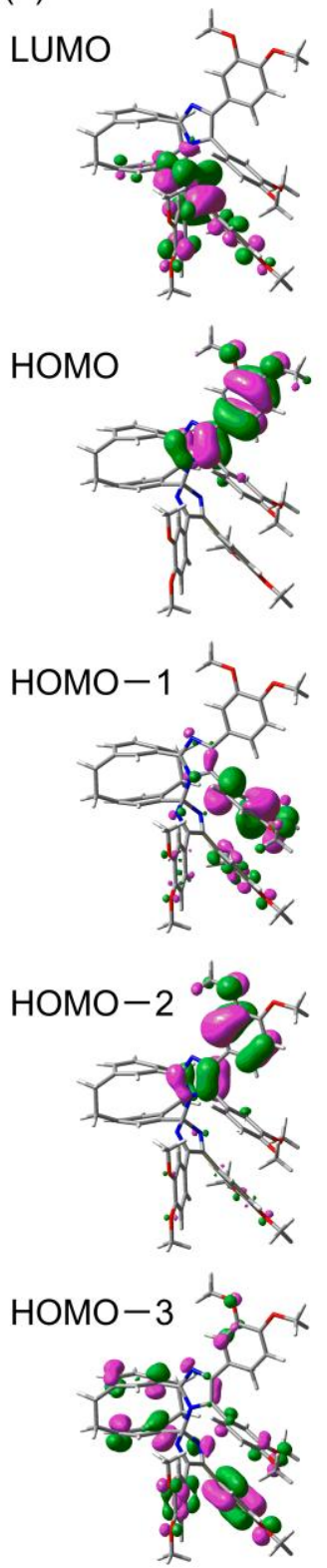

Figure 2. The relevant molecular orbitals of (a) pseudogem-bisDPI[2.2]PC and (b) pseudogem-bisTMDPI[2.2]PC obtained at the MPW1PW91/6-31+G(d) level.

extinction coefficient must form around the UVA radiation region. It is notable that the two imidazole rings- $\operatorname{Im} 1$ and $\operatorname{Im} 2$ - are not equivalent in their electronic environment. We decided that either the introduction of electron-donating groups into the phenyl rings attached to $\operatorname{Im} 2$ or the introduction of electron-withdrawing groups into the phenyl rings attached to Im1 would give rise to the CT transition with a pronounced $f$ value, because of the large orbital overlap between the MO delocalized over the imidazole ring and that

delocalized over the substituted phenyl rings. Since the photoinduced homolytic bond cleavage of the $\mathrm{C}-\mathrm{N}$ bond results from the $\mathrm{S}_{1}$ state [8c], essentially a consequence of the electronic transition from the HOMO to the LUMO, the CT transition from electron-donating substituent groups to $\operatorname{Im} 2$ is considered to be more effective for photochromic reaction.

We designed and synthesized pseudogembisTMDPI[2.2]PC, composed of two 3,3', 4,4'-tetramethoxydiphenylimidazoles (TMDPI) with electron-donating tetramethoxy-substituted phenyl rings. As can be seen in Fig. 1(b), pseudogem-bisTMDPI[2.2]PC shows relatively strong absorption band in the UVA radiation region, as expected. As with the transition for pseudogem-bisDPI[2.2]PC, the $\mathrm{S}_{0} \rightarrow \mathrm{S}_{1}$ transition at $500 \mathrm{~nm}$ demonstrated by the TDDFT calculation also has small oscillator strength $(f=$ $0.001)$ and is described by a HOMO $\rightarrow$ LUMO transition. The TDDFT calculation gives an additional band $\left(\mathrm{S}_{0} \rightarrow \mathrm{S}_{3}\right)$ at $366 \mathrm{~nm}$ with $f=$ 0.1339 . This transition can be attributed to the intramolecular CT transition from tetramethoxy-substituted phenyl rings to $\operatorname{Im} 2$, since the major contribution of this transition is HOMO $-3 \rightarrow$ LUMO. The HOMO-3 is delocalized over the tetramethoxy-substituted phenyl rings. Thus, we can form a CT transition band with a large extinction coefficient in the solar UVA radiation region for the [2.2]PC-bridged imidazole dimer. This strategy of molecular design for increasing photosensitivity is very versatile, and a wide variety of electron-donating substituent groups can be applied to control the photosensitivity as required.

Pseudogem-bisTMDPI[2.2]PC undergoes photochromic reaction involving a color change from no color to green upon UV light irradiation, both in the solid and in solution at room temperature. Under continuous irradiation, the solution reaches photostationary equilibrium very quickly, and after irradiation ceases, the absorption decreases very rapidly according to monoexponential thermal-bleaching kinetics. In contrast to the photochromic behavior of pseudogem-bisDPI[2.2]PC, pseudogem-bis TMDPI[2.2]PC shows photochromic color change even under sunlight, as expected from the UV-vis absorption spectrum. Fig. 3(a) shows the transient vis-NIR absorption spectra of pseudogem-bisTMDPI[2.2]PC in benzene at 
(a)

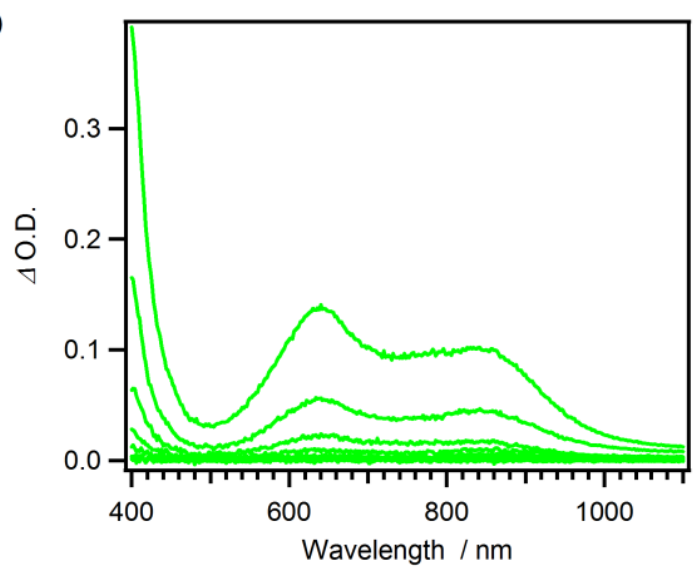

(b)

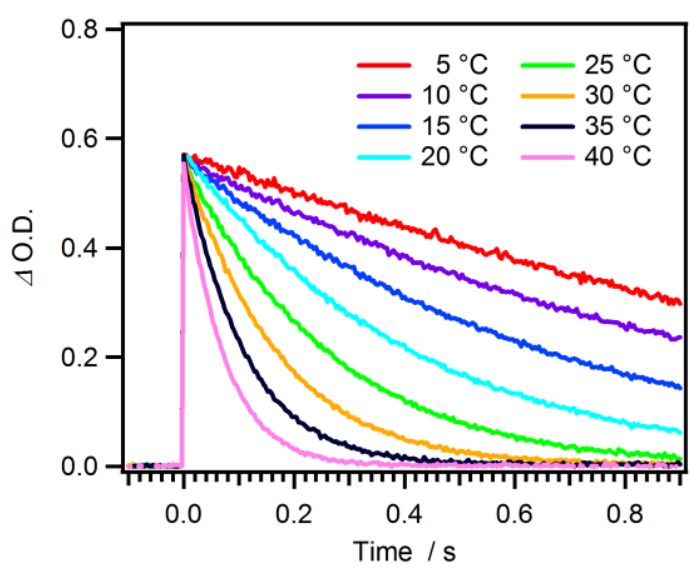

Figure 3. (a) Transient vis-NIR absorption spectra of pseudogem-bisTMDPI[2.2]PC in degassed benzene at $25{ }^{\circ} \mathrm{C}\left(2.1 \times 10^{-4} \mathrm{M}\right.$, light-path length: $\left.10 \mathrm{~mm}\right)$. Each of the spectra was recorded at $200 \mathrm{~ms}$ intervals after excitation with a nanosecond laser pulse (excitation wavelength, $355 \mathrm{~nm}$; pulse width, 5ns; power, 8 $\mathrm{mJ} /$ pulse). (b) Decay profiles of the colored species generated from pseudogem-bisTMDPI[2.2]PC, monitored at $400 \mathrm{~nm}$ in degassed benzene $\left(2.1 \times 10^{-4} \mathrm{M}\right)$. The measurements were performed in the temperature range from $5{ }^{\circ} \mathrm{C}$ to $40^{\circ} \mathrm{C}$.

$25{ }^{\circ} \mathrm{C}$, excited with a ns laser pulse at $355 \mathrm{~nm}$. A sharp absorption band at $400 \mathrm{~nm}$ and a broad absorption band ranging from 500 to $1000 \mathrm{~nm}$ can be ascribed to the colored biradical, pseudogem-bisTMDPIR[2.2]PC. Indeed, the transient absorption spectra are almost identical to those for pseudogem-bisDPI[2.2]PC. The half-life of the colored species is $173 \mathrm{~ms}$ at $25^{\circ} \mathrm{C}$. Fig. 3(b) shows the time profiles of the transient absorbance at $400 \mathrm{~nm}$, measured at temperatures ranging from 5 to $40{ }^{\circ} \mathrm{C}$. The temperature dependence of the half-life of pseudogembisTMDPIR[2.2]PC in benzene solution are summarized in Fig. 4. The enthalpies and entropies of activation $\left(\Delta H^{\star}\right.$ and $\Delta S^{\star}$, respectively) for the thermal back-reaction were

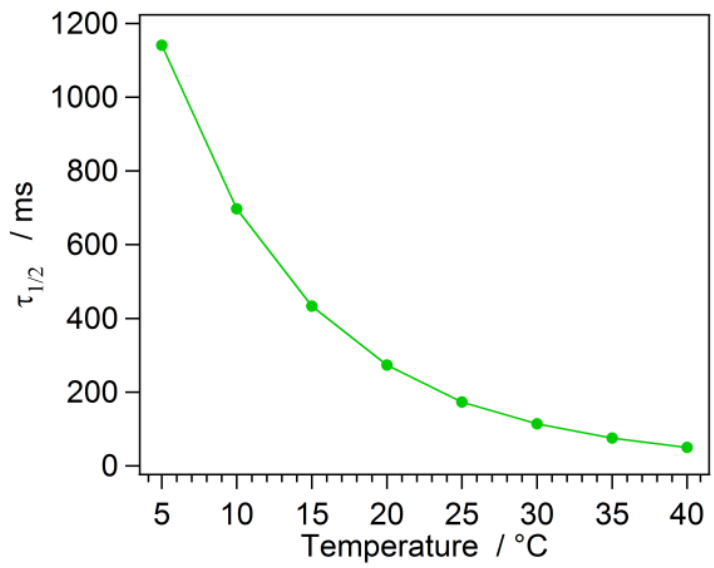

Figure 4. Temperature dependence of the half-life of the colored species generated from pseudogem-bisTMDPI[2.2]PC in degassed benzene solution $\left(2.1 \times 10^{-4} \quad \mathrm{M}\right)$ determined from the measurements of the nanosecond laser flash photolysis experiment.

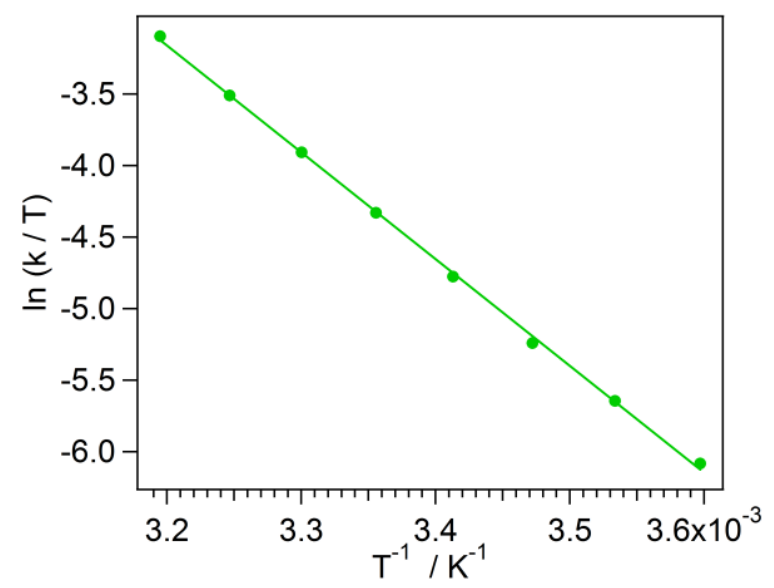

Figure 5. Eyring plots for the thermal back-reaction of the colored species generated from pseudogem-bisTMDPI[2.2]PC in degassed benzene solution $\left(2.1 \times 10^{-4} \mathrm{M}\right)$.

estimated from Eyring plots over temperatures ranging from 5 to $40{ }^{\circ} \mathrm{C}$ (Fig. 5). The Eyring plots produce an excellent straight line, and the $\Delta H^{*}$ and $\Delta S^{\ddagger}$ values estimated from standard least-squares analysis of the Eyring plots are 62.1 $\mathrm{kJ} \mathrm{mol}^{-1}$ and $-25.2 \mathrm{~J} \mathrm{~K}^{-1} \mathrm{~mol}^{-1}$, respectively. The free energy barrier $\left(\Delta G^{\ddagger}=\Delta H^{\sharp}-\mathrm{T} \Delta S^{\ddagger}\right)$ is $69.6 \mathrm{~kJ}$ $\mathrm{mol}^{-1}$ at $25^{\circ} \mathrm{C}$.

\section{Conclusions}

The present study demonstrates that the photosensitivity of [2.2]PC-bridged imidazole dimers, which show instantaneous coloration upon exposure to UV light and rapid fading in the dark, can be readily tuned through appropriate 
design of the phenyl rings attached to the imidazole rings. It is also found that UV-vis absorption spectra can be predictable from the TDDFT (MPW1PW91/6-31+G(d)) calculation. The diversity of the molecular design and the ease of synthesis make this class of photochromic molecules highly attractive for high-performance ophthalmic plastic lenses and revolutionary optical switching devices. The potential for controlling other properties, such as the thermal-bleaching rate and coloring, is important and should be the focus of future investigations.

\section{Acknowledgement}

This work was supported by a Grant-in-Aid for Scientific Research in a Priority Area "New Frontiers in Photochromism (No. 471)" from the Ministry of Education, Culture, Sports, Science and Technology (MEXT), Japan and by a High-Tech Research Center project for private universities with the matching fund subsidy from MEXT.

\section{Reference}

1) a) Organic Photochromic and Thermochromic Compounds, J. C. Crano, R. J. Guglielmetti, Ed., Plenum Press, New York, (1999). b) Photochromism: Molecules and Systems, $\mathrm{H}$. Dürr, H. Bouas-Laurent, Ed., Elsevier, Amsterdam, (2003). c) K. Amimoto,T. Kawato, J. Photochem. Photobiol. C: Photochem. Rev. 6 (2005) 207.

2) a) M. Irie, T. Fukaminato, T. Sasaki, N. Tamai, T. Kawai, Nature 420 (2002) 759. b) Molecular Switches, B. L. Feringa, Ed., Wiley-VCH, Weinheim, Germany, (2001). c) A. Fernandez-Acebes, J.-M. Lehn, Adv. Mater. 10 (1998) 1519. d) F. M. Raymo, M. Tomasulo, Chem. Eur. J. 12 (2006) 3186.

3) a) M. Tomasulo, S. Sortino, A. J. P. White, F. M. Raymo, J. Org. Chem. 70 (2005) 8180. b) M. Tomasulo, S. Sortino, F. M. Raymo, Org. Lett. 7 (2005) 1109. c) S. Jockusch, N. J. Turro, F. R. Blackburn, J. Phys. Chem. A 106 (2002) 9236. d) N. Malic, J. A. Campbell, R. A. Evans, Macromolecules 41 (2008) 1206. e) L. Raboin, M. Matheron, J. Biteau, T. Gacoin, J. P. Boilot, J. Mater. Chem. 18 (2008) 3242. f) S. Kawai, T. Nakashima, K. Atsumi, T. Sakai, M. Harigai, Y. Imamoto, H. Kamikubo, M. Kataoka, T. Kawai, Chem. Mater. 19 (2007) 3479.

4) Y. Kishimoto, J. Abe, J. Am. Chem. Soc. 131
(2009) 4227.

5) A. Sengul, H. Arslan, H. S. Bayari, O. Buyukgungor, Struct. Chem. 19 (2008) 467.

6) a) M. Psiorz, R. Schmid, Chem. Ber. 120 (1987) 1825. b) H. Zitt, I. Dix, H. Hopf, P. G. Jones, Eur. J. Org., Chem. (2002) 2298. c) V. E. Sergeeva, I. V. Rozenberg, Y. D. Antonov, V. E. Vorontsov, A. Z. Starikova, V. I. Fedyanin, H. Hopf, Chem. Eur. J. 11 (2005) 6944.

7) Gaussian 03, Revision E.01, M. J. Frisch, G. W. Trucks, H. B. Schlegel, G. E. Scuseria, M. A. Robb, J. R. Cheeseman, J. A. Montgomery, Jr., T. Vreven, K. N. Kudin, J. C. Burant, J. M. Millam, S. S. Iyengar, J. Tomasi, V. Barone, B. Mennucci, M. Cossi, G. Scalmani, N. Rega, G. A. Petersson, H. Nakatsuji, M. Hada, M. Ehara, K. Toyota, R. Fukuda, J. Hasegawa, M. Ishida, T. Nakajima, Y. Honda, O. Kitao, H. Nakai, M. Klene, X. Li, J. E. Knox, H. P. Hratchian, J. B. Cross, V. Bakken, C. Adamo, J. Jaramillo, R. Gomperts, R. E. Stratmann, O. Yazyev, A. J. Austin, R. Cammi, C. Pomelli, J. W. Ochterski, P. Y. Ayala, K. Morokuma, G. A. Voth, P. Salvador, J. J. Dannenberg, V. G. Zakrzewski, S. Dapprich, A. D. Daniels, M. C. Strain, O. Farkas, D. K. Malick, A. D. Rabuck, K. Raghavachari, J. B. Foresman, J. V. Ortiz, Q. Cui, A. G. Baboul, S. Clifford, J. Cioslowski, B. B. Stefanov, G. Liu, A. Liashenko, P. Piskorz, I. Komaromi, R. L. Martin, D. J. Fox, T. Keith, M. A. Al-Laham, C. Y. Peng, A. Nanayakkara, M. Challacombe, P. M. W. Gill, B. Johnson, W. Chen, M. W. Wong, C. Gonzalez, J. A. Pople, Gaussian, Inc., Wallingford CT (2004).

8) a) T. Hayashi, K. Maeda, Bull. Chem. Soc. Jpn. 33 (1960) 565. b) A. Kimoto, S. Niitsu, F. Iwahori, J. Abe, New J. Chem. 33 (2009) 1339 c) H. Miyasaka, Y. Satoh, Y. Ishibashi, S. Ito, Y. Nagasawa, S. Taniguchi, H. Chosrowjan, N. Mataga, D. Kato, A. Kikuchi, J. Abe, J. Am. Chem. Soc. 131 (2009) 7256. d) K. Fujita, S. Hatano, D. Kato, J. Abe, Org. Lett. 10 (2008) 3105. e) S. Hatano, J. Abe, J. Phys. Chem. A 112 (2008) 6098. f) F. Iwahori, S. Hatano, J. Abe, J. Phys. Org. Chem. 20 (2007) 857. g) A. Kikuchi, F. Iwahori, J. Abe, J. Am. Chem. Soc. 126 (2004) 6526. h) J. Abe, T. Sano, M. Kawano, Y. Ohashi, M. M. Matsushita, T. Iyoda, Angew. Chem. Int. Ed. 40 (2001) 580. i) M. Kawano, T. Sano, J. Abe, Y. Ohashi, J. Am. Chem. Soc. 121 (1999) 8106. 